\title{
Editorials
}

\section{Thyroid hormone replacement in the preconception period and pregnancy}

\section{INTRODUCTION}

Hypothyroidism is common in women of reproductive age and occurs in 2-3\% of all pregnancies. ${ }^{1,2}$ Suboptimal thyroid function in pregnancy carries significant risks of poor obstetric outcomes including pregnancy loss and offspring neurodevelopmental impairment. $^{2}$ These outcomes could potentially be improved through preconception care targeted at women of reproductive age with hypothyroidism. Most women with hypothyroidism are managed in general practice, and facilities for annual thyroid monitoring, medication reviews, and the maintenance of hypothyroidism disease registers, are available within existing primary care resources. In addition, the vast majority of women with hypothyroidism who become pregnant have pre-existing thyroid disease and are already established on treatment by the time of conception. Thus, opportunities abound in general practice to optimise thyroid hormone replacement and prepare women with thyroid dysfunction for conception. In this editorial, the authors discuss the challenges of preconception management of hypothyroidism and highlight current recommendations and a new patient education resource.

\section{CHALLENGES OF THYROID HORMONE REPLACEMENT IN PREGNANCY}

Thyroid hormones are essential for normal fetal growth and development. ${ }^{2}$ The devastating neurological consequences of severe iodine deficiency or neglected congenital hypothyroidism in children are now thankfully rare but remain grim reminders of the importance of thyroid hormones for fetal development. More subtle effects of thyroid hormone deficiency are widespread. Landmark studies in the 1990s showed that children born to women with uncorrected hypothyroidism during pregnancy had a 7-point IQ deficit compared to children of euthyroid mothers. ${ }^{3}$ Since then, numerous observational studies have shown increased risks of pregnancy loss and child neuro-intellectual impairment in untreated, as well as suboptimally treated, women with hypothyroidism. ${ }^{2,4}$ An adequate supply of thyroid hormones is particularly important in early gestation as the fetus cannot synthesise thyroid hormones until the second trimester, and before this time, relies wholly on maternal thyroxine sources. ${ }^{5}$ For women without a functioning thyroid gland or with impaired thyroid reserve, these demands must be met by an increase in levothyroxine dose, failure of which threatens the survival of the fetus. ${ }^{5}$

In practice it is challenging to achieve adequate gestational thyroid function in women with hypothyroidism. About $30-60 \%$ of levothyroxine-treated women have suboptimal thyroid function in early pregnancy, including a proportion who remain overtly hypothyroid through pregnancy. ${ }^{4}$ The reasons for this are practical as well as physiological. Hypothyroid women generally require a $25-50 \%$ increase in levothyroxine dose on conception ${ }^{1}$ but dose adjustments are often inadequate, absent, or instituted late. ${ }^{6}$ Furthermore, early pregnancy may be accompanied by vomiting, treatment nonadherence, or the use of antacids and iron preparations, all of which reduce thyroid hormone absorption or availability. From a practical perspective, only half of pregnancies in the general population are planned ${ }^{7}$ and pregnancy may not be recognised until mid-gestation. More so, access to specialist antenatal clinic services is often delayed, with an average initial evaluation time of about 14 weeks gestation for women with hypothyroidism. ${ }^{6}$ Prior to this time care responsibilities may be blurred, and women may find themselves caught, without monitoring, between primary and secondary care services.

\section{PRECONCEPTION COUNSELLING}

A thyroid disease preconception strategy that is coherent and feasible within present care structures is paramount. ${ }^{5}$ Preconception care is cost-effective and has been shown to improve pregnancy outcomes in women with other chronic disease states, such as diabetes and epilepsy. ${ }^{8}$ Recent guidelines of the American Thyroid Association (ATA) now place increasing emphasis on preconception optimisation of thyroid function in women with thyroid disease. ${ }^{1}$ The goal of preconception thyroid management is to optimise thyroid function and educate patients in preparation for conception. The ATA guidelines recommend that women with primary hypothyroidism who are planning pregnancy should have their levothyroxine dose adjusted to maintain a thyroidstimulating hormone (TSH) concentration $<2.5 \mathrm{mu} / \mathrm{L}$ at the time of conception. ${ }^{1}$ This threshold applies to women with established hypothyroidism and should not be confused with the diagnostic threshold which should remain the same as the non-pregnant population reference interval.

\section{PATIENT EDUCATION}

Clear, consistent, and practical information should be provided to all women of reproductive age, including those who are not imminently planning pregnancy. Education should be addressed ideally at the time levothyroxine treatment is initiated or reissued, and opportunistically during routine clinical encounters. A systematic approach through annual and structured medication reviews would be another efficient approach. Women should be reassured that satisfactory pregnancy outcomes are achievable with adequate thyroid hormone replacement and should be counselled on treatment adherence, correct levothyroxine administration (for example, on empty stomach, with water, at least half an hour before food, beverages, or other medications), and the need to optimise thyroid hormone replacement before conception. Other relevant risk factors such as smoking, alcohol, diet, and adequate iodine intake should be addressed.

\section{THE BRITISH THYROID FOUNDATION THYROID AND PREGNANCY GUIDANCE}

The British Thyroid Foundation (BTF), a leading UK patient charity, have recently launched thyroid and pregnancy guidance for patients, and a thyroid and pregnancy patient alert card which summarises relevant preconception advice from currently available guidelines. UK national thyroid guidelines are still under 
development, and until these are available, current recommendations are based on the ATA Guidelines. ${ }^{1}$ The BTF guidance has been endorsed by the British Thyroid Association and the British Association of Endocrine and Thyroid Surgeons. This valuable resource can be downloaded and printed for patients during the consultation or attached to their levothyroxine prescription and should help empower women of reproductive age with hypothyroidism to take a lead in obtaining blood tests, instituting dose changes, and arranging reviews with their GP on conception. In another initiative, an electronic protocol with alerts to remind GPs to check and optimise thyroid function in individuals with thyroid disease can be incorporated into clinical management systems. ${ }^{10}$ The protocol was shown to be successful in improving thyroid monitoring and also had modest effects on the adequacy of thyroid hormone replacement. ${ }^{10}$ Additional input in terms of patient and clinician education will be needed to accompany regular monitoring. Similar dedicated alerts to remind clinicians to offer written thyroid and pregnancy advice to women of reproductive age who are receiving levothyroxine treatment may be another useful strategy and are currently being trialled.

\section{CONCEPTION ADVICE}

On conception, levothyroxine dose should be increased to mirror the post-conception increase in thyroid hormone requirements. ${ }^{1} \mathrm{~A}$ rule of thumb is to increase levothyroxine dose by $25 \mathrm{mcg}$ daily in women receiving doses of $\leq 100 \mathrm{mcg}$, and by $50 \mathrm{mcg}$ daily in women receiving $>100 \mathrm{mcg}$ daily. ${ }^{2,5}$ Alternatively, the weekly dose can be increased by doubling the dose on 2 days a week, equivalent to a $30 \%$ increase. ' Specific instructions should be provided to women to:

1. increase levothyroxine dose on confirmation of pregnancy;

2. obtain a thyroid blood test; and

3. arrange to review the blood test results with a healthcare practitioner as soon as possible.

Time is of the essence, and specialist endocrine advice needs to be sought as soon as possible when pregnancy is confirmed, rather than wait until the first antenatal appointment. Women on alternative treatments, such as liothyronine (T3) or desiccated thyroid preparations, should be switched to levothyroxine under specialist endocrine guidance as the safety of these preparations is not established in pregnancy. ${ }^{1}$
Lastly, it is essential that facilities are in place for seamless transition of care following conception. Local arrangements will vary but should include rapid access to laboratory services and telephone or e-mail advice from specialists. Primary and secondary care collaborations will be vital in organising transitional care structures and in setting future research and audit priorities.

\section{CONCLUSION}

The discourse on improving thyroid disease outcomes in pregnancy has traditionally been confined to endocrinologistsand obstetricians. However, primary care is often the first point of contact for patients at the time pregnancy is confirmed, and correct management in early pregnancy is essential in ensuring optimal outcome. Among thyroidologists, and within the public at large, a huge volume of debate has centred on issues such as the significance of borderline thyroid dysfunction or the role of alternative thyroid therapies like T3. Relevant as they are, these controversies should not eclipse the pressing challenges of improving preconception care in women with established hypothyroidism. These challenges can be addressed in primary care through patient education and effective preconception optimisation of levothyroxine therapy, as highlighted in the BTF thyroid and pregnancy patient guidance. ${ }^{9}$ To summarise, women with hypothyroidism who are planning pregnancy should have their levothyroxine dose adjusted to maintain a preconception TSH concentration $<2.5 \mathrm{mu} / \mathrm{L}$. On conception, the daily dose of levothyroxine should be increased by $25-50 \mathrm{mcg}$ and thyroid function should be monitored to ensure TSH remains $<2.5 \mathrm{mU} / \mathrm{L}$. Lastly, seamless transfer of care to specialist services should be initiated as soon as possible on conception.

\section{Anh Tran,}

GP, Shadbolt Park House Surgery, Worcester Park and Longcroft Clinic, Banstead.

\section{Steve Hyer,}

Consultant Endocrinologist, Epsom and St Helier University Hospitals NHS Trust, Carshalton.

\section{Imran Rafi,}

Senior Lecturer in Primary Care Education, Institute of Medical and BioMedical Education (IMBE), St George's University of London, London.

\section{Onyebuchi Okosieme,}

Consultant Endocrinologist, Prince Charles Hospital, Cwm Taf University Health Board, Merthyr Tydfil.

\section{Provenance}

Freely submitted; externally peer reviewed.

\section{ADDRESS FOR CORRESPONDENCE}

Onyebuchi Okosieme

Prince Charles Hospital, Cwm Taf University Health Board, Gurnos Road, Merthyr Tydfil CF47 9DT, UK.

Email: OkosiemeOEAcardiff.ac.uk

\section{Competing interests}

Anh Tran, Steve Hyer, and Imran Rafi have no conflicts of interest. Onyebuchi Okosieme is in receipt of a research grant from Concordia International, manufacturers of levothyroxine.

DOI: https://doi.org/10.3399/bjgp19X703805

\section{REFERENCES}

1. Alexander EK, Pearce EN, Brent GA, et al. 2017 Guidelines of the American Thyroid Association for the Diagnosis and Management of Thyroid Disease During Pregnancy and the Postpartum. Thyroid 2017; 27(3): 315-389.

2. Chan S, Boelaert K. Optimal management of hypothyroidism, hypothyroxinaemia and euthyroid TPO antibody positivity preconception and in pregnancy. Clin Endocrinol (Oxf) 2015; 82(3): 313-326.

3. Haddow JE, Palomaki GE, Allan WC, et al. Maternal thyroid deficiency during pregnancy and subsequent neuropsychological development of the child. N Engl J Med 1999: 341(8): 549-555.

4. Taylor PN, Minassian C, Rehman A, et al. TSH levels and risk of miscarriage in women on long-term levothyroxine: a community-based study. J Clin Endocrinol Metab 2014; 99(10): 3895-3902.

5. Okosieme OE, Khan I, Taylor PN. Preconception management of thyroid dysfunction. Clin Endocrinol (Oxf) 2018; 89(3): 269-279.

6. Khan I, Witczak JK, Hadjieconomou S Okosieme OE. Preconception thyroidstimulating hormone and pregnancy outcomes in women with hypothyroidism. Endocr Pract 2013; 19(4): 656-662.

7. Wellings $\mathrm{K}$, Jones $\mathrm{KG}$, Mercer $\mathrm{CH}$, et al. The prevalence of unplanned pregnancy and associated factors in Britain: findings from the third National Survey of Sexual Attitudes and Lifestyles (Natsal-3). Lancet 2013; 382(9907): 1807-1816.

8. Lassi ZS, Imam AM, Dean SV, Bhutta ZA. Preconception care: screening and management of chronic disease and promoting psychological health. Reprod Health 2014; 11 Suppl 3: S5.

9. British Thyroid Foundation (BTF). Pregnancy and thyroid disorders - guidance for patients. 2018. http://www.btf-thyroid.org/projects/ pregnancy/375-pregnancy-and-thyroiddisorders-guidance laccessed 30 Apr 2019).

10. Tran A, Hyer S, Rodin A, et al. Improvements in monitoring and biochemical control of hypothyroidism in primary care with the use of an electronic protocol: 12-month follow up evaluation. Endocrine abstracts 2018; 59: 219. 